the U.S. National Space Science Data Center which also functions as a COSPAR World Data Center. The facts of each deposition have been submitted to the IAU Information Bureau on Astronomical Ephemerides as well as to other appropriate offices. These data are openly available from the Data Center."

\title{
WORKING GROUP GEOLOGY AND GEOPHYSICS OF THE MOON
}

Membership: G. Fielder (Chairman), E. Anders, R. B. Baldwin, W. E. Elston, B. Levin, J. O'Keefe, S. K. Runcorn, R. G. Strom.

The goal of the Working Group was formulated as follows:

"The principal aim of the Working Group is to assist with the geological and geophysical interpretation of the Moon, in close connection with astrophysical implications. The Working Group might also wish to make recommendations on the most efficient means of analysing the lunar surface and interior including returned samples."

Upon the invitation of NASA Headquarters, the Working Group was involved in the final selection of the Apollo 16 and 17 landing sites.

Recommendations by the experts of the W.G. were formulated as an introduction in the book The Moon, IAU Symposium no. 47, Newcastle-upon-Tyne, 22-26 March 1971, S. K. Runcorn and H. C. Urey Editors (D. Reidel Pub. Co. 1972).

The Working Group prepared, under the direction of G. Fielder, an important progress report about the scientific results acquired during the past three years on the geology and geophysics of the Moon. This basic document is following.

\section{A review of the scientific results $1969-1972$}

\section{Anomalies in the Moon's elemental abundances}

Spectrometric measurements from the Apollo 15 and 16 command modules of the fluorescent $\mathrm{X}$-rays produced by the interaction of solar X-rays with the Moon's surface have shown that maria have relatively low $\mathrm{A} 1 / \mathrm{Si}$ ratios and the highlands have higher values of this ratio characteristic of plagioclase-rich materials (anorthosites) (Adler et al., 1972). The highland material returned by Luna 20 and Apollo 16 consisted almost entirely of anorthositic rocks: this lent strong support to the hypothesis that the lunar highlands were, in general, of anorthositic composition.

The bulk composition of the Moon is apparently unique among sampled astronomical bodies; it is neither primitive (solar or chondritic) nor Earth-like. By comparison with solar abundances the elements are consistently depleted, relative to silicon, by amounts which depend chiefly on their volatility. No trace of water in free or combined form has been proved in lunar rocks. Carbon and nitrogen are present in abundances of only 1 to $10 \mathrm{ppm}$.

No indigenous or noble gases have been found. The depletion of other elements ranges from two to three orders of magnitude for highly volatile metals such as $\mathrm{Pb}, \mathrm{Bi}$, $\mathrm{Tl}$, to from ten to three-fold for the somewhat less volatile alkali metals $\mathrm{Na}$ and $\mathrm{K}$. The group of noble metals $(\mathrm{Co}, \mathrm{Ni}, \mathrm{Au}, \mathrm{Ir}$, Re, the platinum group, and other metals near the centre of the periodic table) are deficient though by no means volatile. It is generally believed that, on the Earth, these elements were reduced to the metallic form, dissolved in molten iron, and drawn down to form the core. The depletion patterns on Earth and Moon differ, however, gold being more strongly depleted on the Moon by two orders of magnitude. In general the noble metals of the lunar surface rocks have abundances of only $10^{-4}$ to $10^{-5}$ times their solar abundances, implying segregation of a metal phase. Apparently the Earth and Moon lost their noble metals in separate events, under different chemical conditions.

The depletions of volatile elements seem to reflect an initial deficiency (probably fractionation during accretion from the solar nebula) rather than a subsequent loss.

Refractory elements ( $\mathrm{Al}, \mathrm{Ca}, \mathrm{Sr}, \mathrm{Ti}, \mathrm{Zr}$, $\mathrm{Th}$, rare earths) are so abundant in lunar surface rocks that at least their source region of these rocks (upper 200 to $300 \mathrm{~km}$ ) must have been enriched 3 to 10 fold, originally, over solar abundances. 


\section{Lunar geological domains: their rock types and ages}

The lunar highlands seem to be composed chiefly of rocks of an anorthosite-norite-tractolite association (ANT). This is a rock suite which consists mostly of calcic members of the plagioclase feldspar group $\left(\mathrm{CaAl}_{2} \mathrm{Si}_{2} \mathrm{O}_{8}-\mathrm{NaAlSi}_{3} \mathrm{O}_{8}\right)$, with variable amounts of pyroxenes, $(\mathrm{Mg}, \mathrm{Fe}$, $\mathrm{Ca})_{2} \mathrm{Si}_{2} \mathrm{O}_{6}$, (mainly $\mathrm{Mg}$-rich and $\mathrm{Fe}$-poor varieties), and $\mathrm{Mg}$-rich olivines, $(\mathrm{Mg}, \mathrm{Fe})_{2} \mathrm{SiO}_{4}$. Radiometric dates available give ages of actual rocks near $4 \cdot 1 \times 10^{9}$ years for highland material. Calculated model ages are as high as $4.6 \times 10^{9}$ years.

The maria consist of iron-rich basalt and some coarse-grained rocks of the same composition (ferrogabbro) as well as ultra-mafic rocks. In these rocks pyroxene and olivine tend to be more abundant than plagioclase and the pyroxenes are more Fe-rich than those of the highland ANT suite. Ilmenite $\left(\mathrm{FeTiO}_{3}\right)$ is abundant locally, as in the Apollo site 11. These rocks seem to have been erupted between 3.8 and $3.1 \times 10^{9}$ years ago, in floods which spread at least as far as those of terrestrial flood basalts.

Some of the lava flows in Mare Imbrium have been well photographed by Lunar Orbiter and Apollo orbiting spacecraft and these and other flows have been located - mainly in the maria using Earth based ultraviolet and infrared photography. The flows appear to differ greatly in age. The most recent flows in Mare Imbrium have thickness of a few tens of metres. Horizontal layering in the sides of the Hadley Rille is seen to differ in thickness from a few metres to tens of metres (Apollo 15).

Rocks of the Fra Mauro (Apollo 14) site are largely fragmental or glassy. An important component has the composition of norite or high-aluminum-basalt rich in KREEP - an acronym for K, the Rare Earth Elements, and P.It has been suggested that this material represents the liquid line of descent of magma formed during melting in the early history of the Moon, while the ANT highland suite formed as crystal cumulates from the same magma.

There is evidence in the Fra Mauro area for an event at about $8 \times 10^{8}$ years ago - possibly the formation of Copernicus - which distributed some of the noritic particles widely over the lunar surface. In lunar rocks from all sites there are also small amounts of interstitial glass enriched in silica and alkalies, which resemble terrestrial granites or rhyolites in some ways, as well as rocks low in $\mathrm{SiO}_{2}$ and alkalies (enriched in $\mathrm{MgO}$ ).

Tektites differ markedly from lunar norites and granites but, in view of the limited sampling, the lunar volcanic origin of tektites is not excluded ( $O^{\prime}$ Keefe) at the present time.

The anorthositic particles from the Apollonius highlands, sampled by Luna 20, seem to contain substantial amounts of metallic iron grains (Vinogradov et al., 1972). Regardless of interpretation, the ANT and Fra Mauro rocks suggest large-scale melting and magmatic differentiation of at least the outer few hundred $\mathrm{km}$ of the Moon early in lunar history. The high-Fe mare basalts indicate a later period of fractionation and partial melting. To account for the early melting of the highlands requires a revision of concepts of lunar thermal history.

In spite of early magmatic differentiation of the Moon, and in spite of the present-day large thickness of the outer solid layer (crust), the relatively recent (possibly about $10^{9}$ years' ago or less) formation of large craters (for example, Copernicus, Aristarchus, Tycho) both on highlands and maria was accompanied by lava effusions, even at the crests of the rims (Strom and Fielder, 1970, Fielder, 1971). The origin of these liquid lavas remains unknown.

The basin of Mare Imbrium is $3.9 \times 10^{9}$ years old, judging from the dating of the Apollo 14 rocks, assumed to be ejecta from the Imbrian basin. Flooding of Mare Imbrium by lava seems to have occurred much later - about $3.3 \times 10^{9}$ years' ago (Apollo 15). Apollo 12 lavas are as young as $3.16 \times 10^{9}$ years and Apollo 11 lavas date at about $3.7 \times 10^{9}$ years.

The events that are recorded on the lunar surface occurred early in the history of the solar system: the Moon provides data on the early evolution of the solar system, data which have been almost totally obliterated from the Earth's face.

\section{Heat flow and radioactivity}

A single heat flow measurement at Hadley Rille (Apollo 15) confirms earlier Russian Earth-based 
measurements of $35 \mathrm{ergs} \mathrm{cm}^{-2} \mathrm{~s}^{-1}$. This is about three times larger than that expected on the basis of thermal history calculations applied to lunar models, and is half the terrestrial value, but implies several times as much energy in ergs $\mathrm{cm}^{-3}$ throughout the Moon as that in the Earth. This is an argument for a hot lunar interior at great depths; although, if the Moon were convecting, the surface heat flux would have little bearing on the central temperature, which might still be below the melting point.

Measurements from the Apollo 15 command module of $\gamma$-rays emitted by $\mathrm{U}$, Th and $\mathrm{K}$ showed that the concentration of these elements in Mare Imbrium and in the neighbouring lava flows of Oceanus Procellarum is much higher than elsewhere on the Moon. Concentration of Th in Mare Imbrium is $10 \mathrm{ppm}$ by weight - ten times larger than in the eastern part of the Moon. The concentrations of radioactive elements in the Imbrian region are similar to those found in KREEP basalts, which have been found in large quantities at the Aoollo 14 site and in smaller quantities at all other Apollo sites. It is difficult to explain why the Imbrian and Procellarian lavas should be so rich in radioactive elements. At the Hadley Rille site, the temperature at $1 \mathrm{~m}$ depth is about $252^{\circ} \mathrm{K}$ or about $20^{\circ}$ higher than the average over the lunar surface. These results would not be inconsistent with the existence of quite local thermal anomalies on Moon, perhaps connected, with local inhomogeneties in the distribution of radioactive elements.

\section{Structure and figure of Moon}

The recent impact of a large meteorite within the Apollo network of seismometers was an event which could occur normally only once in 100 years. From energy considerations it is reasonable to suppose that this meteoroid formed a crater the size of a football-stadium. The event helped greatly in the interpretation of the Moon's deep structure.

Seismic records of artificial and natural seismic events indicate that the outer part of the Moon is layered and has a fairly sharply bounded crust about $60 \mathrm{~km}$ thick. The crust is divided into an upper section about $20 \mathrm{~km}$ thick, with a velocity of sound similar to that found in mare basalts, and a lower crust, about $40 \mathrm{~km}$ thick, in which the seismic velocity is higher and similar to that in anorthosites or in eclogite. Below the $60 \mathrm{~km}$ crust the seismic velocities are still higher - similar to those in terrestrial olivine-rich rocks. These observations confirm that at least the outer part of the Moon has been differentiated and either completely or partially melted. Parts of the lunar interior seem to be soft now.

At least in the region around Fra Mauro, the crust is capped by a loosely coherent, granular regolith, several metres in depth, lying on a stratified or otherwise structurally heterogeneous layer possibly consisting of fractured rocks - up to $2 \mathrm{~km}$ thick which is responsible for the long duration, and other unusual properties, of the lunar seismic signals.

The value of $C / M a^{2}$ for the Moon, where $C$ is the Moon's polar moment of inertia, $M$ the mass, and $a$ the radius of the Moon, differs by not more than about 1 per cent from that of a homogeneous body. The Moon may or may not have only a small core.

Despite its high thermal output, the Moon's seismic activity is less than that of the Earth by many orders of magnitude. Feeble quakes have been observed to originate at depths of about $800 \mathrm{~km}$ with epicentres apparently below the edges of the maria. This indicates that the lunar interior at these depths is rigid enough to support considerable non-hydrostatic stress. The seismic activity at these centres correlates with the Moon's position in its orbit. Both in space and time, therefore, this phenomenon agrees generally with predictions from studies of bright temporary spots on the Moon. Yet no quake has correlated unmistakably with a bright spot.

Ignoring convection, thermal calculations (Wood, 1972; Toksöz et al., 1972) show that, in spite of the early melting of the Moon, the outer layers down to a depth of several hundred kilometres must be solid (meaning, below its melting point) at the present time. If convection had occurred in the Moon it could have become solid in $10^{8}$ years if it had been totally molten initially. The solidity of at least the outer parts of the Moon is consistent with the existence of seismic sources at depths of $800 \mathrm{~km}$, and of peculiarities in the geometrical form of the lunar equator revealed by laser ranging from the Apollo 15 command module (Wollenhaupt and Sjogren, 1972). Laser ranging 
measurements have shown that the visible hemisphere of the Moon is depressed below the mean sphere described around the centre of mass, whilst the averted hemisphere is raised above this level. This confirms that the centre of mass and the centre of figure of the Moon are separated by a few kilometres.

It is probable that mascons are old enough to have achieved isostatic equilibrium unless the Moon has always been extremely rigid throughout most of its volume. Isostatic equilibrium could exist now in the region of mascons if the Moon had a moderately rigid crust with a density less than that of its interior. This crust needs only be of the order of $100 \mathrm{~km}$ thick. (Hulme, 1972).

\section{The lunar tectonic grid}

There is a network of major lineaments (fractures, faults, and ridges) in evidence all around the Moon's surface (Fielder, in preparation). Fine lineaments, at least on the centrimetric scale, may be a function of lighting conditions, however (Howard and Larsen, 1972). Linear Features (appearing like the outcropping of tipped, bedding planes, but possibly the expression of more nearly vertical fractures) have been seen in the Apennine mountains (Apollo 15). The origin of the Moon's tectonic grid has not been resolved; convection currents, adjustment of the Moon's figure, and tidal stresses have been proposed as agents controlling the form of grid pattern.

The maximum theoretical tensile stress in an idealised lunar crust due to the tidal attraction of the Earth varies between about 1 bar (apogee) and 1.5 bars (perigee). This is insufficient to initiate fracture in homogeneous rock, but could re-mobilise existing cohesionless fractures. In order to fracture homogeneous rock by tidal stressing, the Moon would have to have been within about 20 Earth-radii at some time past. In order to produce north-south oriented principal stressing (the commonly assumed requisite to form the grid system) by the same mechanism, the Moon would also have to have been in free, rather than in synchronous, rotation relative to the Earth (Gash, in preparation).

\section{Magnetic field, electrical conductivity and temperature of the Moon}

Comparison of the varying magnetic field at the Moon's surface with the field in space shows that the field lines are preferentially crowded near the Moon's surface in certain localities. Analyses by different authors (e.g. Sonett et al.; Sill; Dyal and Parkin) of the response of the Moon to variations of the interplanetary magnetic field have not yet converged on a unique lunar conductivity model. However, the variation of the magnetic field indicates a conducting region in the Moon which is usually interpreted as a hot region. The indicated central temperature is certainly not far below $1000^{\circ} \mathrm{C}$. Temperatures up to $1500^{\circ} \mathrm{C}$ appear possible. A particular conductivity profile does not give a unique model of the present-day temperature distribution within the Moon because the electrical conductivity of rocks depends not only on their mineralogical composition but also on minute differences in the oxygen content (Wright, 1971; Housley and Morin, 1972).

Permanent magnetic fields of different magnitudes exist at all Apollo landing sites and, as shown by measurements from the Apollo 15 and 16 sub-satellites, all around the Moon's equatorial zone. They are due to remanent magnetization of nearby subsurface material. Some of this magnetization is regarded by some as thermoremanence acquired at the time of solidification in a field greater than $10^{3} \mathrm{G}$. Attempts to account for such a field (solar or terrestrial field, lunar dynamo, etc.) remain unsuccessful. However, the real nature of the remanent magnetization of lunar rocks is not yet shown (Nagata et al., 1972). In particular, the possible role of impact magnetization (Hide, 1972) has not been studied.

\section{The cratering and origin of the Moon}

The Moon's low iron content, as indicated by its low density, probably reflects preferential accretion of silicate over metal grains from the solar nebula. Analogous fractionations are observed in meteorites (Urey, Larimer and Anders). The strong depletion in noble metals seems to imply segregation of an iron or sulphide phase during the differentiation of the Moon's crust and mantle. 
Absolute ages established by the various lunar landings allow us to develop a coherent picture of the cratering of the Moon throughout geological history. The outer layers of the Moon are observed to have distorted under lunar gravity in a manner which can be explained by the flow of cold rocks, a limiting case of isostatic adjustment. From observations of the degradation and distortion of large craters we can establish a sequence of relative ages, and, by tying these ages into the determinations of the ages of the maria, an absolute time scale - albeit a tentative one - for crater formation has been found.

The largest lunar craters have surrounding rings, both raised and depressed. Two major theories have been advanced to account for these rings. One calls for post-impact subsidence along impactgenerated ring faults whilst the other postulates a giant tsunami caused by the impact. The wave progresses through rock which has been overstressed to the point at which it is temporarily fluidized.

Crater counts made on the floors of large craters which can themselves be assigned approximate ages may be used to estimate the flux of objects falling on the Moon through geological history. It is important to count only primary impact craters, however.

The premare cratering rates yield a half-life of the planetesimals possibly of the order of $10^{8}$ years. The original source of these objects became depleted so rapidly that by the time the flooding of the maria was completed - some $3 \times 10^{9}$ years ago - these bodies were practically gone. Because the rate of infall was greatest at the earliest observable time it is probable that these bodies did not come from the asteroid belt: no method is known of ejecting bodies so quickly, and in the observed quantities, from an asteroid belt of the form presently observed. It is unlikely that the planetesimals could have been Earth-satellites swept up as the Moon moved outward, driven by tidal friction, because the craters in question were formed over some $10^{9}$ years; and the average diameter of the planetesimals was essentially equal to the average distance between their orbits (Baldwin) - a highly unstable configuration. Consequently, an extinct population of asteroidal bodies ('planetesimals'), in orbits of low eccentricity and $a=1 \mathrm{AU}$ has been postulated. Inasmuch as this early source was depleted rather quickly, the post-mare craters on the Moon - and similarly aged impact craters on the Earth - require a different source and this is now assumed to be the comets or the normal asteroid belt.

Post-mare cratering on the Moon ties in very well with the intensive studies of Canadian impact craters and the less well known craters in Europe and elsewhere. Some of the Canadian craters have been cored as well as studied by various geophysical methods such as measures of gravitational and magnetic anomalies and field and laboratory work, so that we now have rather well defined ideas of the mechanics of crater formation.

Idealised stress wave models provide more details of the explosion process than simple models involving the conservation of energy or momentum. When correlated with measurements of the dynamic incompressibility of lunar surface materials, stress wave models yield predictions of the trajectories of fragments which could form secondary craters and of the depth/diameter ratios of primary craters. The results of these calculations can be checked against the appropriate parameters of experimentally produced craters on Earth and Moon. Numerous man-made objects have impacted the Moon, forming craters in the regolith. The crater dimensions allow a determination of the effect of lower gravity on the sealing of craters in loose material (Wilson and Gash, in preparation).

Explosion craters may be generated by either impact or volcanism. Whilst many of the returned samples show evidence of hypervelocity shock, unshocked lunar rocks resemble terrestrial igneous rocks in texture and composition. On Earth, igneous activity finds its surface expression in volcanotectonic features which commonly resemble craters. Since both impact and magmatic processes have been demonstrated, it would be reasonable to suppose that both shaped the surface of the Moon early in its history. Which process predominated, and whether one process obliterated evidence of the other, remains uncertain though the majority of workers favor impact.

The Moon was a geologically active body during the first $1.5 \times 10^{9}$ years of its life, with impacts and volcanism moulding its face. The early, intense bombardment stage may have lasted for more than $0.5 \times 10^{9}$ years. 


\section{Regolith formation and impacts}

The manner of formation of the ubiquitous lunar regolith (soil) is disputed. Most authors favor simple mechanical smashing of rocks and the ballistic transportation of rock particles. But a minority argue that the soil - of the order of 10 metres thick - is emplaced by processes involving fluidisation. In addition to electrostatic transport - if any - there is occasional evidence of a past gas phase which deposited crystals in the cavities of some of the breccias (rocks formed by breaking and recementation of other rocks). Trenching operations and cores have shown that the soil is layered, and in one core from Apollo 15 this layering seems to have remained undisturbed for $50 \times 10^{6}$ years (Wasserburg). This is consistent with soil transport by impact. At a given location, the regolith grows by successive deposition of thin ejecta layers from small, nearby impacts, but is eventually stripped by a rare, direct hit. The soil particles have a dark amorphous coating, produced by radiation damage (Maurette) and in some cases perhaps by condensation from the vapor phase. O'Keefe has argued that the soil is formed by fluidised flow - base surges deriving from a few large impacts - rather than by ballistic transport and mechanical turnover by a large number of small impacts. He cites the frequency of micro-meteoritic impacts in support of this idea, but the fact that all soil particles contain solar wind gases rules out this possibility. All soil particles must once have been at the very surface, in order to become charged with gas.

There is chemical (but not mineralogical) evidence for a micro-meteorite contamination of only about $1.5 \%$ in the lunar soil, in addition to an ancient component in highland breccias. This component seems to represent debris of planetesimals that bombarded the highland crust in the first $700 \times 10^{6}$ years of the Moon's history (Morgan et al., 1972). Again neither of the two principal rock types (anorthositic gabbros and mare basalts) is like any known meteorite. However, there is evidence of shock metamorphism in surface rocks. It is suspected that impact cannot launch significant numbers of macroparticles at the velocity of escape from the Moon $\left(2.3 \mathrm{~km} \mathrm{~s}^{-1}\right)$, and small craters can be used, in principle, to determine relative ages of geological units not saturated with craters. For a given geological unit it is necessary, however, to eliminate secondary craters from the count and then to estimate the ratio of internally to externally produced craters - an operation that can be attempted statistically (Fielder et al., 1972) - before making comparisons between geological units of the number-densities of what are, specifically, primary impact craters.

\section{Outstanding questions in lunar science}

Since all integrations (non-convective models) indicate that the Moon's heat is at present confined to the deep interior, it follows that recent volcanic rocks might well be the most significant goals for future exploration.

Problems still outstanding are (i) the deep internal structure of the Moon; in particular, does the Moon have a small, heavy core; and is it solid or liquid? (ii) did the Moon once have a strong magnetic field? (iii) the composition of the deep interior, (iv) has the Moon ever suffered convection? (v) the origin of numerous craters - particularly, the major highland craters - and other features such as rilles, (vi) the importance of impact-generated and other types of volcanism, (vii) the history of the Moon earlier than $4 \times 10^{9}$ years' ago, and (viii) the origin of the Moon itself. These and other problems can only be resolved by concerted effort of the international scientific community.

The numerous data stemming from the manned and unmanned lunar exploration programmes of the U.S.A. and U.S.S.R. are being studied fairly intensively but there is still room for more adequate studies - particularly those of an interdisciplinary nature. Funding for research on the backlog of data now accessible to students of the Moon should be made available to them by all nations. 